\title{
Lipid profile in pre-eclampsia in comparison with normal subjects
}

\author{
Misra M.K ${ }^{1}$, Tiu $\mathbf{D N}^{2}$, Sharma $\mathbf{R}^{3}$ \\ ${ }^{1}$ Dr. Manish Kumar Misra, Associate Professor, Department of Biochemistry, ${ }^{2}$ Dr. Devendra Nath Tiu, Associate \\ Professor, Department of Physiology, ${ }^{3}$ Dr. Rina Sharma, Professor, Department of Obstetrics \& Gynaecology, all authors \\ are affiliated with Mayo Institute of Medical Sciences, Barabanki, U.P, India.
}

Address for Correspondence: Dr. Manish Kumar Misra, Email: manishreena2751@ rediffmail.com, 2/33, Vikas Khand, Gomti Nagar, Lucknow, UP, India

\begin{abstract}
Introduction: The present study was designed to evaluate the association of dyslipidemia (abnormal lipid metabolism) in pre-eclampsia in comparison with non pregnant women, normotensive pregnant women and post partum subjects. Material ad Methods: This study was conducted on 200 subjects who were further divided in four groups (50 subjects in each group). The four groups were non pregnant subjects, normotensive pregnant subjects, pre-eclamptic patients and post partum subjects. Each serum sample from different groups was evaluated for total cholesterol (mg/dl), HDLcholesterol (mg/dl), LDL-cholesterol (mg/dl), VLDL-cholesterol (mg/dl) and triglyceride (mg/dl). Result: On comparison of non pregnant subjects, normotensive pregnant subjects and post partum subjects with pre-eclamptic patients, the levels of total cholesterol, LDL cholesterol, VLDL cholesterol and triglycerides were found to be significantly increased ( $p$ values $\leq 0.001$ ) and that of HDL cholesterol levels were found to be statistically significantly ( $p$ values $\leq 0.001$ ) in pre-eclamptic patients. Conclusion: Deranged lipid metabolism in pre-eclampsia is associated with development of atherosclerotic diseases which increases the morbidity and mortality in mother as well as foetus. The various factors responsible for development of dyslipidemia and its prevention need to be studied and evaluated.
\end{abstract}

Key words: Dyslipidemia, Lipid profile, Pre-eclampsia

\section{Introduction}

Pre-eclampsia is one of the most common complications of pregnancy especially in developing countries. Pre-eclampsia is a multisystem disorder of pregnancy characterized by hypertension, proteinuria and oedema. This condition generally remits after delivery. It is a leading cause of maternal and foetal morbidity and mortality [1]. The foetal risk includes intrauterine growth restriction, premature delivery with complications and death. The mother is at risk of renal failure, pulmonary oedema, seizures leading to eclampsia, stroke and finally death. Preeclampsia occurs in $7-10 \%$ of pregnancies worldwide [2]. In India, the national incidence is reported to be $8-10 \%$ of pregnancies. If not treated properly it may lead to eclampsia which is characterized by malignant hypertension and epileptic convulsions requiring emergency caesarean section. Pre-eclampsia is most

Manuscript received $27^{\text {th }}$ September 2016

Reviewed: $11^{\text {th }}$ October 2016

Author Corrected: $21^{\text {st }}$ October 2016

Accepted for Publication 31 $1^{\text {st }}$ October 2016 common during last trimester of pregnancy. Women with family history of essential hypertension are more prone to develop pre-eclampsia. It has been also observed that pre-eclampsia is also associated with metabolic syndrome. There are many hypotheses regarding pathogenesis of pre-eclampsia but the most accepted is theory of oxidative stress. Abnormal serum lipid parameters may lead to oxidative stress in preeclampsia. The risk of pre-eclampsia is increased in women with elevated levels of oxidized low density lipoproteins and triglycerides [3]. Women with a history of pre-eclampsia have significant differences in lipid parameters (dyslipidemia) and an increased susceptibility to lipoprotein oxidation when compared with women who had normal pregnancy. Disorders of lipoprotein metabolism are reported to be a major cause of hypertension and proteinuria in pre-eclampsia. This study is conducted to study the changes in lipid profile (Cholesterol, triglycerides, HDL-cholesterol, LDLcholesterol and VLDL cholesterol) that develops during 
normal pregnancy, in pre-eclampsia and after delivery (post partum).

Despite considerable research, the cause or causes of pre-eclampsia remain unclear and there are no clinically useful screening tests to identify women in whom it will develop.

\section{Materials and Methods}

The present study was conducted on pregnant women and non pregnant women attending or admitted in the Obstetrics and Gynecology Department of Mayo Institute of Medical Sciences, Barabanki, U.P. Total 200 subjects between the age 20- 35 yrs were included in this study which were further subdivided into four groups:-

$>$ Group A-Non pregnant normotensive women.

$>$ Group B- Pregnant women without pre-eclampsia during third trimester of pregnancy.

$>$ Group C-Pregnant women with mild/severe preeclampsia during third trimester of pregnancy (Blood pressure $140 / 90 \mathrm{mmHg}$, with proteinuria, with or without pathological oedema).

$>$ Group D-Post partum pre-eclamptic women (15 or more days after delivery).

Exclusion Criteria: Patients with chronic hypertension, diabetes mellitus, hepatitis, renal disorders, thyroid disease, collagen vascular disease and anaemia were excluded from this study. This study also excludes patients previously treated for Poly Cystic Ovarian Disease and pre-eclampsia /eclampsia.

Sample Collection and Analysis- Samples from all the four study groups were collected from antecubital vein after taking all aseptic measures. After the clot has retracted the sample was centrifuged for 5 minutes, the serum thus obtained was stored at $4^{0} \mathrm{C}$ for lipid profile assessment. In our study we estimated total cholesterol $(\mathrm{mg} / \mathrm{dl})$, HDL cholesterol $(\mathrm{mg} / \mathrm{dl})$, LDL cholesterol $(\mathrm{mg} / \mathrm{dl})$, VLDL cholesterol $(\mathrm{mg} / \mathrm{dl})$ and serum triglycerides $(\mathrm{mg} / \mathrm{dl})$.

All the lipid profile parameters included in this study were analyzed by enzymatic methods.

Frederickson-Friedwald`s formula was used to calculate Serum LDL cholesterol:-

LDL cholesterol $=$ Total cholesterol $-($ HDL cholesterol + VLDL cholesterol).

VLDL cholesterol (VLDL) was calculated as 1/5 of Triglycerides

Statistical analysis was performed with the Chi-square, student $\mathrm{t}$ test. The results were summarised as Mean \pm S.D. and the result values of $\mathrm{P} \leq 0.05$ were considered significant and values of $\mathrm{P} \geq 0.05$ were considered as non-significant.

\section{Result}

According to the table when we compared the total cholesterol levels between all the four groups it was observed that the values are increasing gradually in normotensive pregnant women and pre-eclamptic women as compared with normal subjects and the values are highly significant ( $\mathrm{p}$ values are $\leq 0.0001$ ). The values of total cholesterol are decreasing gradually in post partum women. Again the values are highly significant ( $\mathrm{p}$ values are $\leq 0.0001$ ).

\section{Comparison of lipid profile levels (MEAN \pm S D) in four study group.}

\begin{tabular}{|c|c|c|c|c|}
\hline & Group A & Group B & Group C & Group D \\
\hline Total Cholesterol (mg/dl) & $208.7 \pm 5.2$ & $213.5 \pm 4.3$ & $236.2 \pm 5.9$ & $231.5 \pm 2.7$ \\
\hline HDL Cholesterol (mg/dl) & $43.67 \pm 1.5$ & $58.90 \pm 0.98$ & $39.95 \pm 1.3$ & $41.37 \pm 1.1$ \\
\hline LDL Cholesterol (mg/dl) & $114.21 \pm 5.1$ & $122.34 \pm 4.8$ & $147.64 \pm 4.2$ & $129.34 \pm 3.7$ \\
\hline VLDL Cholesterol (mg/dl) & $26.56 \pm 1.4$ & $44.85 \pm 1.3$ & $57.72 \pm 0.82$ & $48.14 \pm 1.7$ \\
\hline Triglycerides (mg/dl) & $135.5 \pm 5.2$ & $228.6 \pm 6.4$ & $287.1 \pm 3.7$ & $233.4 \pm 2.4$ \\
\hline
\end{tabular}

p values between different groups in relation with the table-From the above table in case of total cholesterol on comparing Group A with Group B, Group C and Group D the values were significantly high and the p values were found to be statistically significant $(\leq 0.001)$. On comparison of Group B with Group C and Group D the $p$ values were found to be statistically significant $(\leq 0.001)$. On comparison of Group $\mathrm{C}$ with Group $\mathrm{D}$ again the values were statistically significant $(\leq 0.001)$. 
Similar results were found on comparison of LDL cholesterol, VLDL cholesterol and triglyceride levels. ( $p$ values $(\leq$ $0.001)$.

In case of HDL cholesterol on comparison of different groups the values are low in preeclampsia, the $\mathrm{p}$ values were found to be statistically significant $(\leq 0.001)$ which again increases again in post partum stage.

On comparing HDL Cholesterol levels between all the four groups, there is significant increase in HDL Cholesterol levels in normal pregnant women compared to non pregnant women. But the levels in pre-eclamptic women are significantly low as compared with non pregnant and normotensive pregnant women. The low levels of HDL Cholesterol in pre-eclamptic subjects may be a reason of atherosclerosis and related disorders in pre-eclampsia. The values of HDL Cholesterol again began to rise in postpartm subjects. These values are statistically significant. ( $p$ values are $\leq 0.0001$ ).

When we compare the LDL cholesterol values between the four groups, it was observed that LDL cholesterol values increases significantly in normotensive pregnant women and preeclamptic women as compared with nonpregnant women ( $\mathrm{p}$ values are $\leq 0.0001$ ). The LDL cholesterol values again started falling during post partum period.

According to the table the VLDL cholesterol and triglyceride values in all the four groups showed same pattern as shown by total cholesterol and LDL cholesterol. The values increased significantly in normotensive pregnant women and preeclamptic women as compared with nonpregnant women and falls after delivery (post partum) [4].

\section{Discussion}

In this study, conducted over a period of one year, all the four groups were comparable in terms of age. Abnormal lipid metabolism (dyslipidemia) plays very important role in the pathogenesis of pre-eclampsia [4]. Increased lipid levels may be associated with endothelial cell dysfunction. In endothelial cell linoleic acid is mainly responsbile for oxidative stress. The serum lipoprotein levels increases during pregnancy and these levels increases upto two folds in preeclamptic patients.Serum triglyceride concentration levels rises more significantly in preeclampsia [5]. It may be due to insulin resistance, hyperlipidemia and up-regulation of inflammatory markers.In pregnancy the estrogen levels are very high and this hyperestrogenemia is responsible for hypertriglyceridemia. The increased levels of triglyceride is related with hypercoagulability [6]. The increase in plasma triglyceride concentration correlates with increase in plasma concentration of small dense LDL [7].

In present study all the lipid parameters (total cholesterol, LDL cholesterol, VLDL cholesterol and triglycerides) except HDL cholesterol were significantly increased as compared with non pregnant women and normotensive pregnant females (i.e. pregnancy without pre-eclampsia). The levels of these parameters were decreased after delivery (post partum). This dyslipidemia may further lead to atherosclerosis which increases the mortality of pre-eclamptic patients. The concentration of HDL cholesterol was decreased in pre- eclamptic women and again we saw an increasing trend in post partum stage [8]. This HDL cholesterol is involved in exchange of cholesterol, cholesterol esters and triglycerides from peripheral tissues to the liver (reverse cholesterol transport) where they are metabolised and excreted. It is also involved in activating lipoprotein which releases fatty acids that can be oxidised by $\beta$ oxidation pathway to provide energy.

Low levels of HDL-Cholesterol may compromise the function of all these processes. In this study HDL cholesterol levels were significantly decreased in preeclamptic women as compared with non pregnant and normotensive pregnant women. The levels were found to be increased in post partum women.

These results are consistent with other studies. So it is evident that dyslipidemia plays a important role in the pathogenesis of pre-eclampsia. The various causative factors leading to dyslipidemia and it's prevention need to be further studied and evaluated.

Funding: Nil, Conflict of interest: None initiated, Permission from IRB: Yes

\section{References}

1. Vanderjagt DJ, Patel RJ, El-Nafaty AU, Melah GS, Crossey MJ, Glew RH. High-density lipoprotein and homocysteine levels correlate inversely in preeclamptic women in northern Nigeria. Acta Obstet Gynecol Scand. 2004 Jun;83(6):536-42. 
2. Mutlu Turkoglu U, Ademoglu E. Imbalance between lipid peroxidation, antioxidant status in preeclampsia. Gynecol Obstet Invest. 1998;46:37-40.

3. Qiu C, Phung TT, Vadachkoria S, Muy-Rivera M, Sanchez SE, Williams MA. Oxidized low-density lipoprotein (Oxidized LDL) and the risk of preeclampsia. Physiol Res. 2006; 55(5): 491-500. Epub 2005 Dec 12.

4. Enquobahrie DA, Williams MA, Butler CL, Frederick IO, Miller RS, Luthy DA. Maternal plasma lipid concentrations in early pregnancy and risk of preeclampsia. Am J Hypertens. 2004 Jul;17(7):574-81.

5. Cekmen MB, Erbagci AB, Balat A, Duman C, Maral $\mathrm{H}$, Ergen $\mathrm{K}$, et al. Plasma lipid and lipoprotein concentrations in pregnancy induced hypertension. Clin. Biochem. 2003; 36(7): 575-8.
6. Kokia E, Barkai G, Reichman B, Segal P, Goldman B, Mashiach S. Maternal serum lipid profile in pregnancies complicated by hypertensive disorders. J Perinat Med. 1990;18(6):473-8.

7. Taylor RN, Roberts JM. Endothelial cell dysfunction. In: Linhheimer MD, Roberts JM, Cunningham FG, eds. Chesley's Hypertensive Disorders in Pregnancy. 2nd ed. Stanford, CT: Appleton \& Lange; 1999: 395-429.

8.Adiga U, D'souza V, Kamath A, Mangalore N. Antioxidant activity and lipid peroxidation in preeclampsia. J Chin Med Assoc. 2007 Oct; 70 (10): 435-8.

9. Gohil J.T. et al, Estimation of Lipid Profile in Subjects of Preeclampsia, The Journal of Obstetrics and Gynecology of India (July-August 2011) 61 (4): 399-403

\section{How to cite this article?}

Misra M.K, Tiu DN, Sharma R. Lipid profile in pre-eclampsia in comparison with normal subjects. Int J Med Res Rev 2016;4(10):1859-1862.doi:10.17511/ijmrr. 2016.i10.24. 